\title{
Correção de distorções harmônicas em sistemas elétricos através de interferência destrutiva
}

\author{
Correction of harmonic distortions in electrical systems through destructive interference \\ Giovani Teixeira de Azevedo ${ }^{1}$, Estaner Claro Romão ${ }^{1}$, Carlos Renato Menegatti*1] \\ ${ }^{1}$ Universidade de São Paulo, Escola de Engenharia de Lorena, Lorena, SP, Brasil
}

\begin{abstract}
Recebido em 04 de Outubro, 2018. Revisado em 17 de Dezembro, 2018. Aceito em 18 de Dezembro, 2018.
\end{abstract}
\begin{abstract}
Neste trabalho será apresentado um método didático e alternativo para correção das distorções harmônicas em sistemas elétricos de corrente alternada. Primeiramente será apresentado o problema das distorções harmônicas e como quantificá-las através da série de Fourier. Em seguida, métodos experimentais para a correção das distorções serão apresentados de forma didática visando associar conceitos básicos de física e ferramentas matemáticas para quantificação do problema. Para medir as taxas de distorção harmônica, antes e depois da correção, foi utilizado o espectro de FFT (Fast Fourier Transform), demonstrando sua aplicação. Os resultados irão demonstrar que as atividades experimentais tiveram como principal destaque a redução da TDH (Taxa de Distorção Harmônica) de $71 \%$ para $11 \%$ utilizando apenas interferência destrutiva.
\end{abstract}

Palavras-chave: correção de distorções harmônicas, interferência destrutiva, formas de onda, série de Fourier.

In this paper, we will present a didactic and alternative method for correction of harmonic distortions in a.c. electric systems. First, the harmonic distortions problem and quantification through the Fourier series will be presented. Then, experimental methods for the correction of distortions will be presented in didactic way aiming to associate basic concepts of physics and mathematical tools to quantify the problem. To measure the harmonic distortion rates, before and after the correction, the FFT (Fast Fourier Transform) spectrum was used, demonstrating its application. The results will demonstrate that experimental activities had as main highlight the reduction of TDH (Harmonic Distortion Rate) from $71 \%$ to $11 \%$ using only destructive interference.

Keywords: correction of harmonic distortions, destructive interference, waveforms, Fourier series.

\section{Introdução}

A forma de onda da tensão elétrica fornecida aos consumidores, em geral, pode apresentar deformações conhecidas como distorções harmônicas. Quando freqüências com múltiplos inteiro da freqüência do sistema de distribuição (50 ou 60 Hertz) surgem e são combinadas com o formato fundamental tem-se o efeito de distorção harmônica na rede de distribuição elétrica $[1,2]$.

Em todos os setores existem equipamentos elétricos ligados à rede, dentre eles os maiores causadores das distorções harmônicas são os de cargas não lineares [3]. Por não aproveitar a energia em seu formato senoidal de onda, esses dispositivos distorcem o sinal da rede, isso ocorre porque eles apresentam uma relação não linear entre a corrente e a tensão deixando alguns resíduos harmônicos no sistema. Dentre os maiores causadores das distorções harmônicas podemos citar: transformadores, motores e sistemas eletrônicos de chaveamento que necessitam trabalhar com a mudança da corrente alternada para corrente contínua.

Esta deformação da forma de onda da tensão na rede acarreta muitas desvantagens a curto e longo prazo [4].

*Endereço de correspondência: renatomenegatti@usp.br
No curto prazo podem causar: sobrecarga, vibrações e envelhecimento de transformadores, indutores e motores. Com a soma da componente harmônica de terceira ordem, as quais, em geral, são mais recorrentes em sistemas elétricos de cargas não lineares, cargas monofásicas podem sobrecarregar os condutores neutros; sobrecarregar e causar envelhecimento de capacitores de compensação de energia reativa; causar perturbações nas redes de comunicação ou das linhas telefônicas. Em longo prazo a presença de harmônicas também pode causar grande prejuízo econômico. Como por exemplo: envelhecimento precoce de cabos e componentes eletrônicos, sendo necessária a substituição prematura; sobrecarga na rede e disparos intempestivos acarretando em paradas dos setores de produção.

Entender as origens, analisar e quantificar as distorções harmônicas se faz extremamente necessário para então encontrar a solução para sua redução [5-7]. Estão presentes no mercado empresas que se empenham em propor soluções para redução das distorções harmônicas. Basicamente há duas estratégias para atenuação dos efeitos das distorções harmônicas, a primeira se trata de aceitar essas distorções sobredimensionando os dispositivos, e a segunda é trabalhar para eliminá-los do sistema. 
No primeiro caso em que se deseja aceitá-las, uma das soluções seria aumentar as potências nominais de fontes e/ou a seção transversal dos cabos. Isso reduziria a fonte de impedância que por sua vez diminuiria a perda por efeito Joule. Já a segunda seria retirá-las parcialmente do sistema, utilizando filtros passivos sintonizados, transformadores especiais ou mesmo indutores a montante das cargas não lineares. Diante desses problemas, pensar em formas de reduzir as distorções harmônicas se faz de extrema valia.

Neste artigo apresenta-se um método alternativo para o estudo e correção de distorções com uso de interferência destrutiva. O método será apresentado de forma didática com o objetivo de associar conceitos básicos de física e ferramentas matemáticas para quantificação do problema. As formas de onda da tensão elétrica serão produzidas por um kit experimental didático de baixo custo e as medidas serão realizadas com osciloscópio. Para quantificação das taxas de distorção harmônica, antes e depois das correções, serão medidos os espectros de FFT demonstrando na prática a aplicação da análise de Fourier. Os resultados serão apresentados através de atividades experimentais.

\section{Considerações teóricas}

Em geral, a maioria dos dispositivos não lineares produz distorções harmônicas ímpares sendo que a maior componente de distorção é dada pelo $3^{\circ}$ harmônico. Este fato é algo intrínseco da maioria dos sistemas elétricos. Já as harmônicas pares, que produzem assimetrias de meiaonda, não são muito comuns nos dispositivos elétricos, mesmo nos casos não lineares. As harmônicas pares se manifestam em menor intensidade em sistemas elétricos do que as harmônicas ímpares e em geral estão relacionadas ao mau funcionamento das cargas não lineares.

A fim de entender e quantificar o problema de distorção na rede se faz necessário a análise harmônica clássica, conhecida como análise de Fourier. A partir dessa ferramenta é possível quantificar o grau de distorção harmônica presente na tensão elétrica [8]. Na análise de Fourier uma função periódica não senoidal pode ser representada por uma série trigonométrica composta pela somatória de senos e ou cossenos. Para maior clareza, na Fig. 1a, 1b e 1c tem-se respectivamente uma representação do modo fundamental do $3^{\circ}$ e $5^{\circ}$ harmônicos. Estas três ondas quando somadas geram a forma de onda distorcida da Fig. 1d.

Dessa forma pode-se afirmar que a forma de onda total (Fig. 1d) é a composição espectral dos $1^{\circ}, 3^{\circ} \mathrm{e}$ $5^{\circ}$ harmônicos. Assim, de maneira geral, representa-se matematicamente a forma de onda da tensão da rede com a função periódica:

$$
\begin{aligned}
V(t) & =V_{1} \operatorname{sen}\left(1 \omega t+\varphi_{1}\right)+V_{2} \operatorname{sen}\left(2 \omega t+\varphi_{2}\right)+\ldots \\
& +V_{n} \operatorname{sen}\left(n \omega t+\varphi_{n}\right)
\end{aligned}
$$

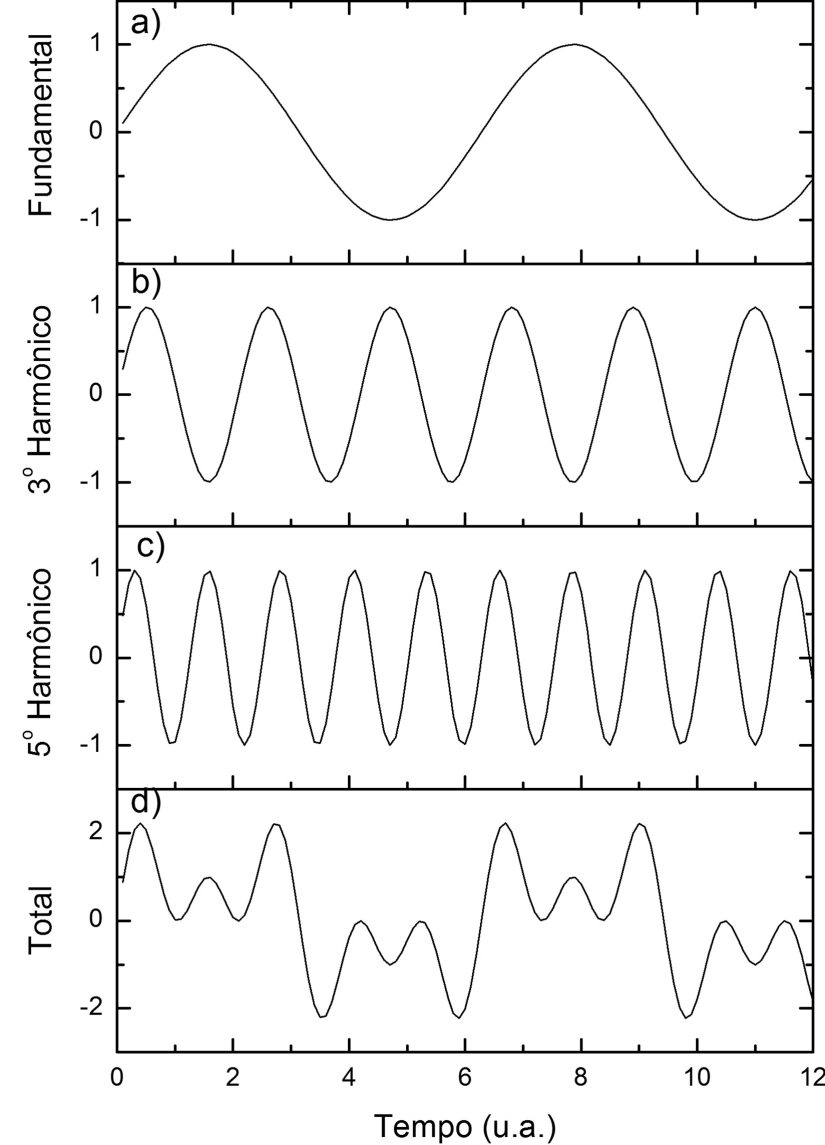

Figura 1: Representação do a) modo fundamental, b) $3^{\circ}$ harmônico, c) $5^{\circ}$ harmônico e d) onda distorcida causada pela somatória dos modos.

Determinando-se os valores das amplitudesdos harmônicos, o cálculo da Taxa de Distorção Harmônica é definido como:

$$
T H D=\frac{\sqrt{\sum_{2}^{n} V_{i}^{2}}}{V_{1}} \times 100
$$

em que $V_{1}$ é o valor de amplitude do modo fundamental e $V_{i}$ a amplitude subsequente de cada harmônico. $\mathrm{O}$ valor da THD é expresso em porcentagem (\%).

A proposta desse trabalho é reduzir a distorção harmônica causada pelo harmônico em específico através de interferência destrutiva. Ou seja, parte-se da premissa que a forma de onda possa ser escrita como seu modo fundamental somada ao harmônico específico, no caso o terceiro harmônico $(\mathrm{n}=3)$, de amplitude $V_{3}$ :

$$
V_{a n}(t) \cong V_{1} \operatorname{sen}(\omega t)+V_{3} \operatorname{sen}(3 \omega t)
$$

Do mesmo modo, pode-se produzir outra tensão com a mesma forma de onda porém defasada por um ângulo $\beta$ :

$$
V_{c n}(t) \cong V_{1} \operatorname{sen}(\omega t-\beta)+V_{3} \operatorname{sen}(3 \omega t-3 \beta)
$$


E assim, subtraindo-se a Eq. (3) da Eq. (4) tem-se a forma de onda total dada por:

$$
\begin{aligned}
V_{a c}(t) & =V_{1} \operatorname{sen}(\omega t)+V_{3} \operatorname{sen}(3 \omega t) \\
& -V_{1} \operatorname{sen}(\omega t-\beta)-V_{3} \operatorname{sen}(3 \omega t-3 \beta)
\end{aligned}
$$

Desse modo, para se eliminar totalmente o harmônico indesejado, é preciso que o segundo termo cancele com o quarto termo da equação 5. Para isso acontecer basta satisfazer que $3 \beta=360^{\circ}$, ou seja, $\beta=120^{\circ}$.Logo,

$$
V_{a c}(t)=\sqrt{3} V_{1} \operatorname{sen}\left(\omega t+30^{\circ}\right)
$$

O efeito do cancelamento do harmônico, resultado da somatória das ondas, é conhecido como interferência destrutiva. Isso fará comque $V_{a c}(t)$ seja uma onda puramente senoidal livre de distorções. Na próxima seção será detalhado o aparato utilizado para produzir tais ondas distorcidas e defasadas de modo a satisfazer a equação 6.

\section{Atividades experimentais}

\subsection{Aparato experimental}

A plataforma experimental utilizada para geração e observação das distorções harmônicas foi desenvolvida pelo mesmo autor e publicado na Revista Brasileira de Ensino em Física [9]. Basicamente três bobinas de nylon com diâmetro de $15 \mathrm{~mm}$ e comprimento de $30 \mathrm{~mm}$, enroladas com 1000 voltas de fio de cobre esmaltado (AWG 24) são posicionadas ao redor de um rotor defasadas em $120^{\circ}$ como mostra a Fig. 2a. O rotor possui 8 slots nos quais podem ser colocados pequenos imãs de neodímio criando diversas configurações espaciais de campo magnético. Sua rotação é controlada por uma tensão DC aplicada em um pequeno motor de $12 \mathrm{~V}$. Para este trabalho foi utilizada a configuração de 4 imãs com pólos magnéticos alternados igualmente espaçados no rotor. As formas de onda das tensões geradas foram obtidas medindo-se a tensão de uma bobina $V_{a n}$ ou $V_{c n}\left(V_{f a s e}\right)$ e de duas bobinas na configuração estrela $V_{a c}\left(V_{\text {linha }}\right)$. Estas grandezas e a ligação estrela estão explícitas na Fig. 2b. As medidas foram feitas utilizando o osciloscópio digital, fabricante Tektronix, modelo TBS 1202B.

\subsection{Observação da forma de onda induzida}

Utilizando o osciloscópio foi medido o sinal induzido em uma das bobinas $\left(\mathrm{V}_{\mathrm{an}}\right)$ conforme Fig. 2b. Para este experimento a frequência do rotor foi ajustada para aproximadamente $65 \mathrm{~Hz}$ e o sinal pode ser observado na Fig. 3a. Neste resultado notou-se uma forma de onda induzida com amplitudes máximas de aproximadamente 1,5 $\mathrm{V}$, que dependem basicamente da distância dos imãs às bobinas e da frequência de rotação. A principal característica que será estudada é sua forma distorcida. Neste aparato, a distorção é produzida principalmente pela
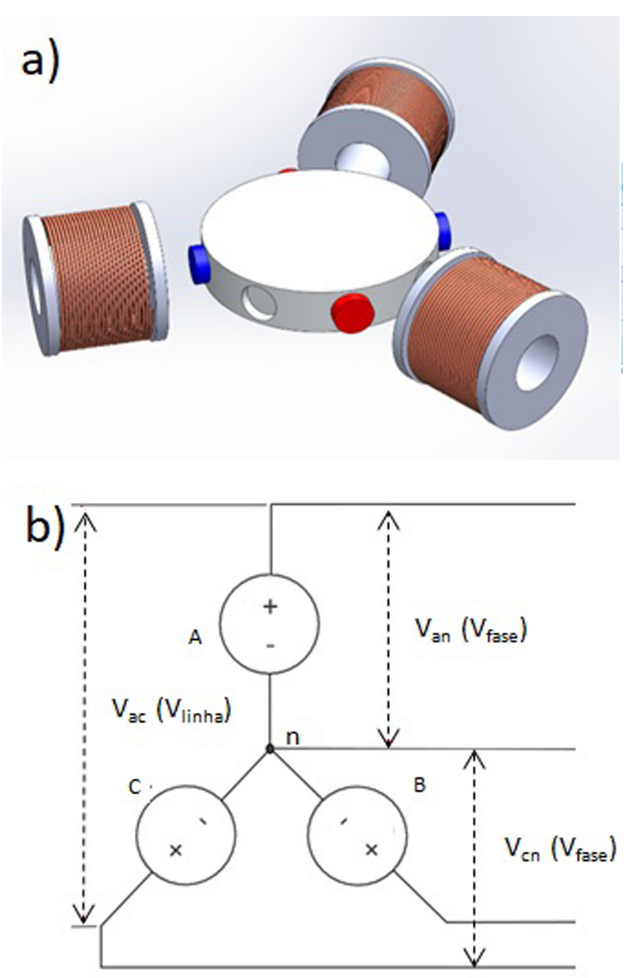

Figura 2: a) Aparato experimental para geração das distorções harmônicas. Bobinas dispostas em ângulo de $120^{\circ}$ ao redor do rotor com 4 imãs de neodímio igualmente espaçados e com pólos magnéticos alternados; b) Esquema de ligação para medida das formas de onda. $V_{\text {an }}$ e $V_{\text {cn }}\left(V_{\text {fases }}\right)$ correspondem as tensões da bobina $A$ e $C, V_{\text {ac }}\left(V_{\text {linha }}\right)$ é a tensão de duas bobinas na configuração estrela.

distribuição espacial do campo magnético criada pelo arranjo específico dos ímãs no rotor (Fig. 2a).

Com o mesmo osciloscópio obteve-se o espectro de Fourier através da função FFT (FastFourier Transform). Esta função permite que o osciloscópio funcione como analisador de espectro decompondo a onda em seus respectivos harmônicos. Na Fig. 3 obtém-se este espectro no qual se observou os principais harmônicos que compõem a forma de onda. $\mathrm{O}$ espectro obtido pelo osciloscópio está na escala $\mathrm{dB}$ na forma $d B=2 \log \left(V_{i} / V_{0}\right)$. Assim, conclui-se que a forma de onda é basicamente composta pelo $1^{\circ}$ harmônico $(\sim 66 \mathrm{~Hz})$, que condiz com a rotação do rotor, $3^{\circ}$ harmônico $(\sim 200 \mathrm{~Hz}), 5^{\circ}$ harmônico $(\sim$ $330 \mathrm{~Hz})$ e $7^{\circ}$ harmônico $(\sim 462 \mathrm{~Hz})$. Os demais picos são causados por ruídos experimentais abaixo de $-35 \mathrm{~dB}$ não sendo considerados harmônicos naturais do sistema. A priori considerou-se apenas as contribuições dos $1^{\circ} \mathrm{e}$ $3^{\circ}$ harmônicos que estão na faixa de -2 e $-5 \mathrm{~dB}$ respectivamente, ou seja, a razão das amplitudes $V_{i} / V_{3}$ é de 1,4. Já os $5^{\circ}$ e $7^{\circ}$ harmônicos ficam em uma faixa abaixo de $-30 \mathrm{~dB}$, ou seja, amplitudes de aproximadamente 20 vezes menor que a fundamental, assim desconsiderou-se suas contribuições por enquanto. Para esta forma de onda foi calculada uma taxa de distorção harmônica de aproximadamente $\mathrm{TDH}=71 \%$. 
a)
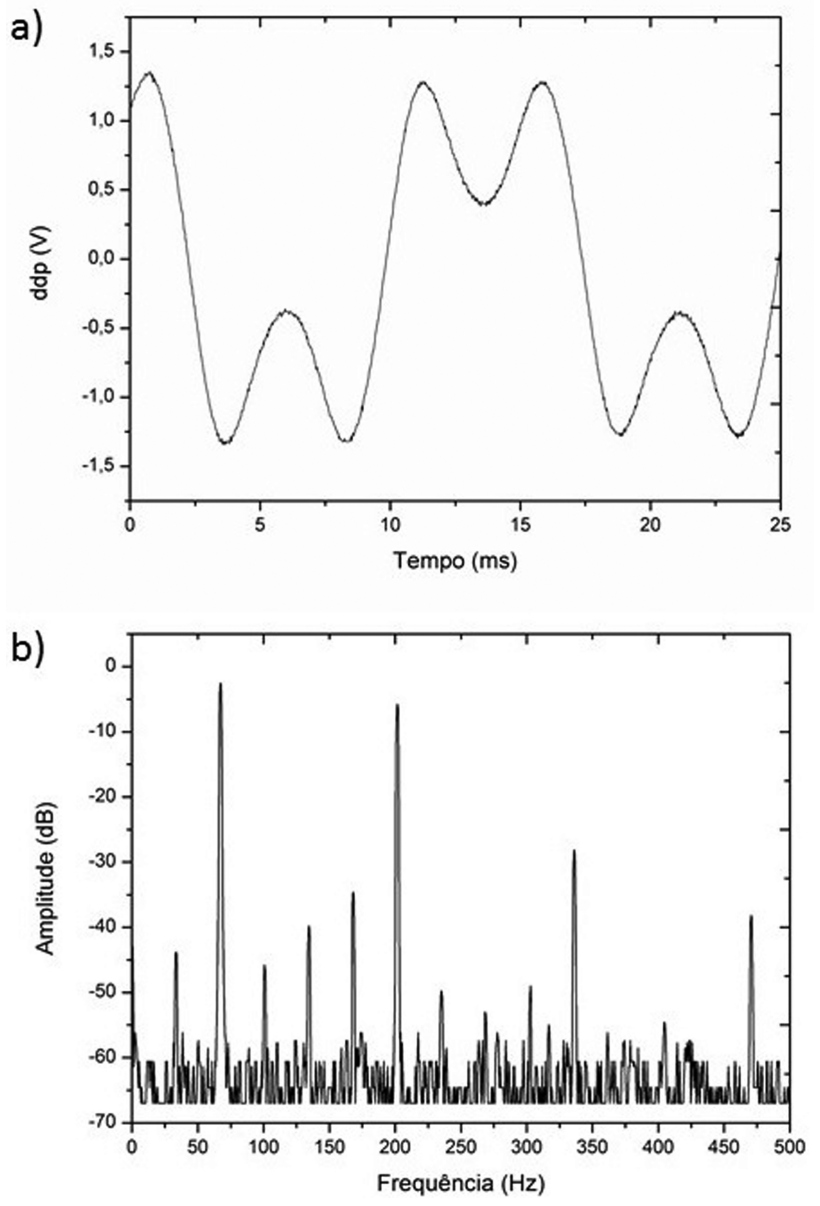

Figura 3: a) Forma de onda distorcida induzida em uma bobina $\left(\mathrm{V}_{\text {fase }}\right)$; b) Espectro de Fourier em dB mostrando os harmônicos constituintes: $1^{\circ}$ harmônico $(\sim 66 \mathrm{~Hz})$, que condiz com a rotação do rotor, $3^{\circ}$ harmônico $(\sim 200 \mathrm{~Hz}), 5^{\circ}$ harmônico $(\sim 330 \mathrm{~Hz})$ e $7^{\circ}$ harmônico $(\sim 462 \mathrm{~Hz})$.

\subsection{Eliminação do $3^{\circ}$ harmônico por interferência destrutiva}

Considerando o espectro obtido na Fig. 3b, foi decomposto a forma de onda $V_{a n}(t)$ produzida por uma bobina como sendo a somatório de seus harmônicos:

$$
\begin{aligned}
V_{a n}(t) & =V_{1} \operatorname{sen}(\omega t)+V_{3} \operatorname{sen}(3 \omega t) \\
& +V_{5} \operatorname{sen}(5 \omega t)+V_{7} \operatorname{sen}(7 \omega t)+\ldots
\end{aligned}
$$

nos quais $V_{1}$ e $V_{3}$ são as amplitudes dos $1^{\circ}$ e $3^{\circ}$ harmônicos enquanto que as amplitudes dos $5^{\circ}$ e $7^{\circ}$ harmônicos serão desprezadas $V_{5}=V_{7}=0$. Considerando que as formas de onda produzidas pelas bobinas são idênticas, assumi-se que a forma de onda total das bobinas em ligação estrela $V_{a c}$ seja descrita conforme equação (6).

Para comprovar o efeito da interferência destrutiva, primeiramente assegura-se que as formas de onda das duas bobinas fossem aproximadamente iguais. Na Fig. 4 tem-se a medida simultânea das tensões de fase de duas bobinas $\left(V_{a n}\right.$ e $\left.V_{c n}\right)$ defasadas em $120^{\circ}$. Nela observa-se que as formas de onda de fato são muito próximas. É

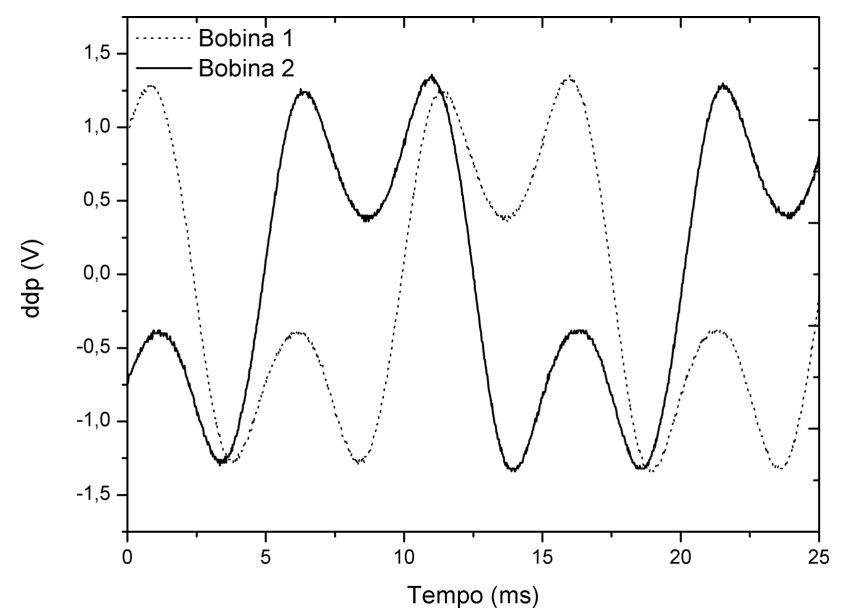

Figura 4: Forma de onda das tensões de fase de duas bobinas $\left(\mathrm{V}_{\text {an }}\right.$ e $\left.\mathrm{V}_{\mathrm{cn}}\right)$ defasadas em $120^{\circ}$.

importante ressaltar que tanto a intensidade do campo magnético de cada imã, geometria e a posição em relação ao rotor das bobinas são fatores que influenciam na forma de onda. Nesta etapa foi feita apenas a análise visual das formas de onda fazendo-se pequenos ajustes no rotor para que ficassem o mais parecidas possível.

Em seguida, associa-se as bobinas em ligação estrela para verificar a interferência destrutiva. A Fig. 5a mostra a medida da tensão de linha $V_{a c}$. Nela observa-se que a forma de onda se aproximou de uma senóide, porém ainda com uma pequena distorção na crista do sinal. A linha pontilhada é um ajuste pela função seno no qual encontra-se um coeficiente de correlação de $\mathrm{R}=$ 0,97 . Na Fig. 5b é apresentada a medida do espectro de Fourier no qual observa-se uma pequena contribuição $\operatorname{dos} 3^{\circ}$ e $5^{\circ}$ harmônicos. Como a amplitude relativa do modo fundamental é de $4 \mathrm{~dB}$ as amplitudes dos $3^{\circ}$ e $5^{\circ}$ harmônicos são aproximadamente 12,5 vezes menores pois estão abaixo de $-18 \mathrm{~dB}$. Desse modo este arranjo entre as bobinas conseguiu reduzir a amplitude do $3^{\circ}$ harmônico pelo fator de 10 levando a taxa de distorção harmônica a aproximadamente TDH $=11 \%$.

\subsection{Eliminação de harmônicosde ordens superiores com filtro passa baixa}

Foi demonstrado na seção 3.3 que a interferência destrutiva reduz drasticamente o $3^{\circ}$ harmônico, isto é confirmado pelo espectro FFT. Entretanto, ainda se percebe uma pequena distorção na onda causada por harmônicos de ordens superiores. Adicionando um filtro passa baixa é possível eliminar esses harmônicos fazendo com que o resultado experimental proposto para a tensão de linha $\left(\mathrm{V}_{\mathrm{ac}}\right)$ seja o mais próximo possível do modelo teórico apresentado na seção 2. O filtro (Fig. 6) foi projetado para a frequência de corte de $300 \mathrm{~Hz}$ de modo a eliminar os $5^{\circ}$ e $7^{\circ}$ harmônicos que poderiam estar causando a pequena distorção na onda. Nas Fig. 7a e 7b apresentam-se 

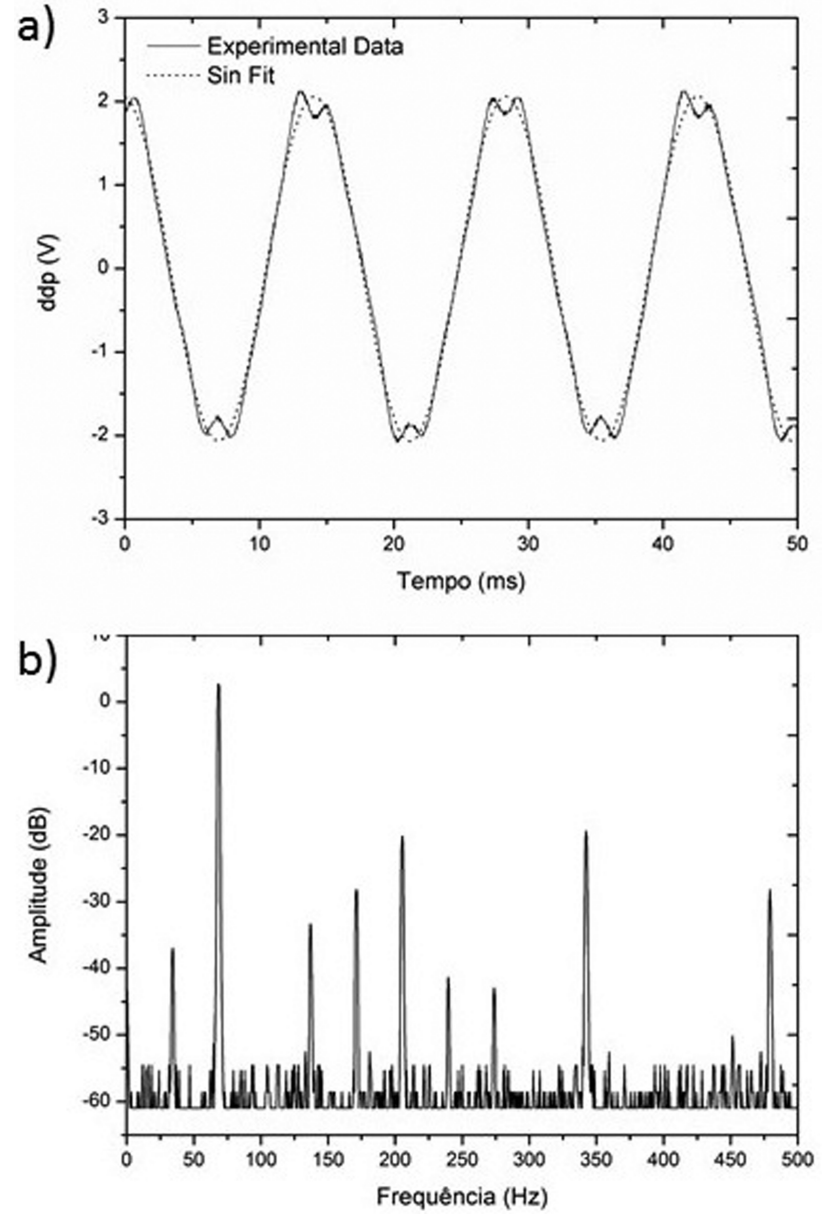

Figura 5: a) Forma de onda da tensão de linha $\mathrm{V}_{\mathrm{ac}}$ gerada por duas bobinas em ligação estrela. A linha pontilha é o ajuste pela função seno. b) Medida do espectro de Fourier. Pode-se observar que as amplitudes dos $3^{\circ}$ e $5^{\circ}$ harmônicos estão abaixo de -18 $\mathrm{dB}$.

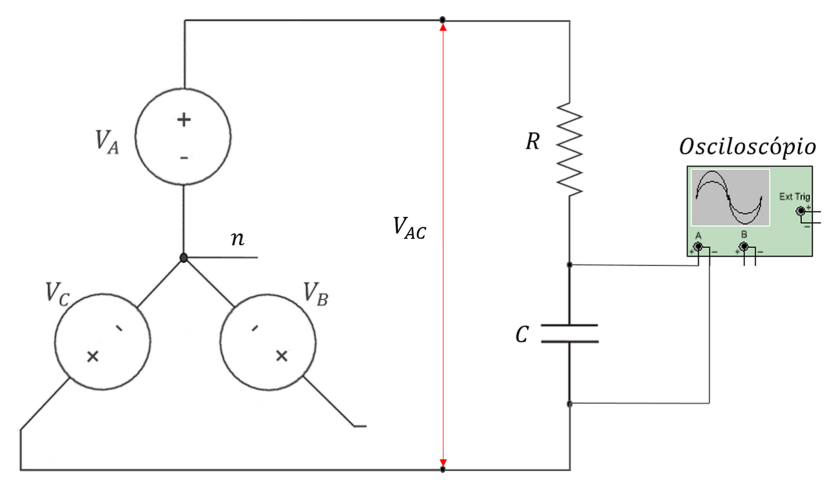

Figura 6: Filtro passa baixa acoplado a tensão de linha $\mathrm{V}_{\mathrm{ac}}$ (este filtro foi projetado com uma frequência de corte de $300 \mathrm{~Hz}$ ).

a forma de onda e o espectro de Fourier respectivamente, medidos conforme montagem da Fig. 6 .

Esse último resultado comprova uma diminuição total da distorção harmônica. Na Fig. 7a observa-se uma forma de onda mais próxima ao formato senoidal. A linha pontilhada é o ajuste pela função seno no qual se encontra
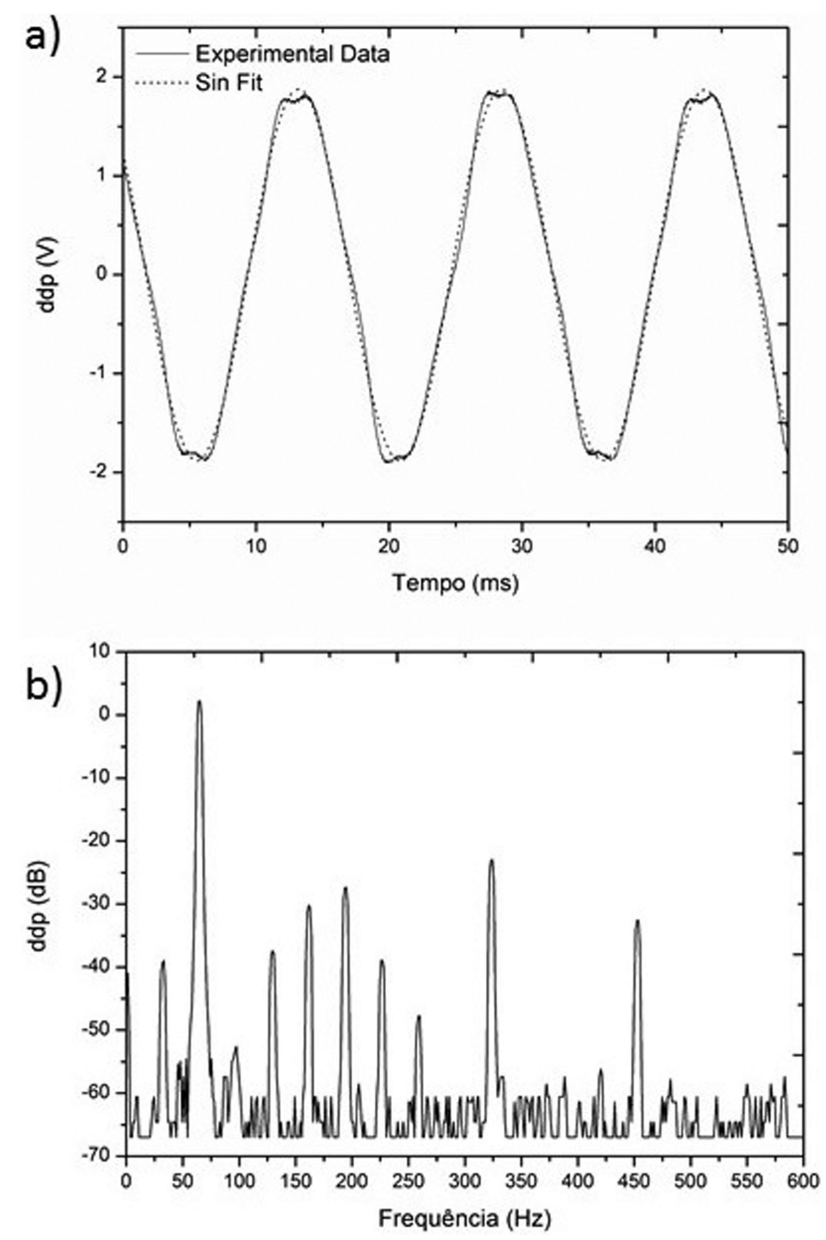

Figura 7: a) Forma de onda da tensão de linha $V_{a c}$ gerada por duas bobinas em ligação estrela acoplada a um filtro passa baixa $\left(f_{\text {corte }}=300 \mathrm{~Hz}\right)$. A linha pontilha é o ajuste pela função seno. b) Medida do espectro de Fourier. Pode-se observar que as amplitudes dos $3^{\circ}$ e $5^{\circ}$ harmônicos foram abaixo de $-24 \mathrm{~dB}$.

o coeficiente de correlação de $\mathrm{R}=0,99$. Na Fig. $7 \mathrm{~b}$ a medida do espectro de Fourier no qual se observa que a amplitude relativa do modo fundamental é de $2,5 \mathrm{~dB}$ e as amplitudes dos $3^{\circ}$ e $5^{\circ}$ harmônicos são aproximadamente 20 vezes menores, pois estão abaixo de $-24 \mathrm{~dB}$. Para esta forma de onda foi calculada $\mathrm{TDH}=6 \%$ comprovando a redução da taxa de distorção harmônica por um fator de 12 vezes.

\section{Conclusões}

Neste trabalho foi apresentado um método didático para compreensão e correção de distorções harmônicas em sistemas elétricos. O problema das distorções harmônicas e seus impactos na rede elétrica foram descritos de maneira geral relevando a importância do desenvolvimento de soluções para reduzí-las. Para sua descrição matemática e quantificação foram utilizados conceitos da série de Fourier. A proposta teórica de correção foi baseada na redução de um harmônico específico, no caso o $3^{\circ}$ 
harmônico, somando-se ondas defasadas criando assim uma interferência destrutiva.

Com relação à parte experimental, foi demonstrado que formas de onda distorcidas podem ser produzidas por efeito de indução magnética por arranjos específicos de magnetos. O aparato experimental foi construído pelo próprio autor, com baixo custo, e já foi descrito em trabalho anterior. Neste trabalho foi produzida uma forma de onda distorcida composta principalmente pelos $1^{\circ}(66 \mathrm{~Hz})$ e $3^{\circ}(200 \mathrm{~Hz})$ harmônicos. Foi mostrado que a forma de onda produzida pode ser facilmente medida com osciloscópio e analisada através da função FFT (Fast Fourier Transform). Esta etapa exige que o estudante adquira certa prática na utilização e interpretação de medidas com osciloscópios.

A solução proposta para redução da distorção harmônica apresentou bons resultados na prática. Foi possível reduzir a TDH de $71 \%$ para $11 \%$ apenas com a interferência destrutiva. Pode-se observar no osciloscópio que a forma de onda se tornou muito próxima de uma onda senoidal quando as formas de ondas distorcidas e defasadas foram associadas em conexão estrela. Na sequência, foi acoplado um filtro RC com frequência de corte de $300 \mathrm{~Hz}$ para eliminar os $5^{\circ}$ e $7^{\circ}$ harmônicos, demonstrando também aplicações de filtros para redução da TDH. Neste caso a TDH foi reduzida para $6 \%$.

Por fim, todos os conceitos teóricos e procedimentos experimentais realizados nesse trabalho podem contribuir como estímulo a alunos, em nível de graduação, no entendimento de diversos conceitos em Matemática, Física e Eletricidade experimental.

\section{Referências}

[1] K.J. Phipps, J.P. Nelson e P.K. Sem, IEEE Trans. on Industry Applications 30, 476 (1994).

[2] J. Arrillaga, D.A. Bradley e P.S. Bodger, Power System Harmonics (John Wiley\&Sons, Nova Jersey, 1989).

[3] L.A. Pomilio e S.M. Deckmann, Eletrônica de Potência 11, 9 (2006).

[4] L.C.E. da Silva, Efeitos das Distorções Harmônicas (Tensões e Correntes) e Desequilíbrios (Tensões) em Medidores Eletrônicos Trifásicos de Energia Elétrica Ativa. Dissertação de Mestrado, Universidade Estadual Paulista, São Paulo (2008).

[5] D.E. Steeper e R.P. Stratford, IEEE Trans. on Industry Applications 12, 232 (1976).

[6] A.L.Z. de Grandi, Metodologia para avaliação de distorção harmônica de corrente em transformadores de distribuição. Dissertação de Mestrado, Universidade de São Paulo, São Paulo (2012).

[7] A.M. Variz, Cálculo do Fluxo de Harmônicas em Sistemas Trifásicos Utilizando o Método de Injeção de Correntes. Tese de Doutorado, Universidade Federal do Rio de Janeiro, Rio de Janeiro (2006).

[8] D.A. Teixeira, Análise das distorções harmônicas - estudo de caso de um sistema industrial. Dissertação de Mestrado, Universidade Federal de Minas Gerais, Belo Horizonte (2009).
[9] G.T. de Azevedo, C.J.T. Peixoto, F. Bargos e C.R. Menegatti, Rev. Bras. Ensino Fís. 39, e3503 (2017). 